\title{
To take risk is to face loss: a tonic pupillometry study
}

\author{
Eldad Yechiam* and Ariel Telpaz \\ Industrial Engineering and Management, Technion - Israel Institute of Technology, Haifa, Israel
}

Edited by:

Itzhak Aharon, The Interdisciplinary

Center, Israel

Reviewed by:

Davide Marchiori, Technion, Israel

Shira Elqayam, De Montfort

University, UK

*Correspondence:

Eldad Yechiam, Faculty of Industrial Engineering and Management,

Technion - Israel Institute of

Technology, Haifa 32000, Israel.

e-mail: yeldad@tx.technion.ac.il
The construct of risk taking is studied through the prism of the relation between tonic arousal and risk taking behavior. Several theories have proposed that high aroused individuals tend to exhibit risk aversion. We posit that this arousal-behavior association is activated much more strongly in risks with losses, as losses increase arousal and trigger relevant traits associated with the sensitivity to risk. In three studies we examined risk taking in experience-based decision tasks, with either token losses or relative-losses (in the gain domain). In Study 1 we found a negative correlation between pre-task pupil diameter and risk taking in the loss domain but not in the gain domain. In Study 2 we re-analyzed a previous pupillometry dataset involving symmetric mixed gains and losses. We found that the negative correlation in this mixed condition emerged even while the participants did not show loss aversion. This finding was replicated in Study 3. Thus, the effect of losses on arousal provides sufficient conditions for the moderation of the tonic arousal-behavior association. The findings suggest an important role for losses in the psychological and physiological experience of risk.

Keywords: risk, arousal, experience, decision, personality, individual differences, pupil

\section{INTRODUCTION}

In this study we examine the relation between losses and the psychophysiological experience of risk. Economic theory traditionally defines risk attitude as the sensitivity to payoff variance (Markowitz, 1952; Pratt, 1964; Sharpe, 1964; and see recent studies which relate brain activity to perceptions of risk as variance, e.g., Preuschoff et al., 2006, 2008). Yet several theories have proposed that losses are somehow implicated in the feeling of risk. For example, Coombs and Lehner $(1981,1984)$ examined a basic lottery where individuals have an equal chance of winning or losing $\$ 10$. They found that adding $\$ 10$ to the loss increased perceived risk more than adding the same amount to the gain, and therefore suggested that the constructs of risk and loss are not independent (see also Duxbury and Summers, 2004). A similar argument is made by attention-based theories of losses, though these theories posit a more general effect of losses on the sensitivity to incentives (Taylor, 1991; Yechiam and Hochman, 2011). Prospect theory (Kahneman and Tversky, 1979) likewise argues that loss aversion is an important component that steers people away from taking risk when it involves gains and losses, because the subjective weight of losses is larger than the subjective weight of equivalent gains. We examine whether losses are an inherent part of what make things risky through the prism of the relation between tonic arousal and risk taking behavior.

Several theories of personality have converged to predict a negative association between tonic arousal and risk taking. In their sensation seeking theory, Zuckerman et al. (1964) suggested that individuals differ in the level of stimulation required for maintaining optimal arousal, with those exhibiting lower levels of arousal requiring more stimulation in order to reach their optimal level of arousal. Since risk taking is one form by which stimulation is achieved, this account suggests that low tonic arousal is associated with a greater tendency to take risk (Farley and Farley, 1972; Ellis, 1987; McNamara and Ballard, 1999) ${ }^{1}$. Eysenck's (1967) personality theory similarly characterized extroverts as low-arousal individuals, who seek stimulating activities in order to heighten their arousal levels (see also Gray, 1987). A somewhat modified account appears in theories of trait anxiety (Zuckerman, 1960; Eysenck, 1992). The literature on trait anxiety suggests that anxious individuals are chronically more aroused on the one hand, and avoid risk and uncertainty on the other (Eysenck, 1992); thus, high arousal is naturally associated with risk aversion. In support of this prediction, studies have shown that high sensation seekers exhibit lower galvanic skin response (GSR) in response to various stimuli (Stelmack et al., 1983; Plouffe and Stelmack, 1986) and even at rest (Gatzke-Kopp et al., 2002).

We propose that the association between tonic arousal and risk taking is moderated by the presence of losses in the experience of risk. It is relatively well known that losses increase arousal and attention (Taylor, 1991; Rozin and Royzman, 2001). Recently, several studies have found that this attentional effect is exhibited independently from loss aversion (Hochman et al., 2010; Hochman and Yechiam, 2011; Yechiam and Telpaz, in press). Based on these findings, we have proposed that this attentional effect of losses provides sufficient conditions for many of the phenomena attributed to losses (Yechiam and Hochman, 2011). For example, it was found that the effect of losses on consistency in risk taking

\footnotetext{
${ }^{1}$ In later writings Zuckerman (1990) maintained the idea of a negative association between arousal level and sensation seeking but focused on the complex interaction between the high reactivity of the dopaminergic system and the weakly reactive serotonergic system.
} 
behavior emerges even in the absence of loss aversion (Yechiam and Telpaz, in press). Similarly, the effect of losses on decision performance was demonstrated even in tasks where usually participants exhibit no loss aversion (Bereby-Meyer and Erev, 1998; Erev et al., 1999). These effects are explained by a mere increase in on-task attention in response to losses.

Here, we extend this argument to suggest that because losses increase arousal and attention more than gains, risks with losses are more "rewarding" for individuals with low tonic arousal. Likewise, risks without losses are less intimidating for those having high arousal. Thus, without losses sensation seekers might not consistently relish and replenish on the risky experience, while anxious people might not be consistently intimidated by it. Therefore, the postulated negative correlation between tonic arousal and risk taking is observed more reliably for risky situations that involve losses. It is further predicted that this effect of losses is not contingent on the decision weight asymmetry induced by losses (i.e., loss aversion; Kahneman and Tversky, 1979). We suggest that the arousal and attention produced by losses are sufficient for producing this effect.

To examine these predictions, we used a pupillometric measure of tonic arousal. The pupil diameter (PD) is an immediate and direct index of autonomic activation (Granholm and Steinhauer, 2004). Changes in PD are controlled by two muscles, the dilator and the sphincter, which are differentially influenced by activity in the sympathetic and parasympathetic branches of the autonomic nervous system. The former channel is mediated primarily by norepinephrine and the latter by acetylcholine (Hutchins and Corbett, 1997). Thus, differently from the GSR, the PD is affected by both branches modulating autonomic arousal, and not only the sympathetic branch. Parasympathetic arousal may be relevant to risk taking behavior since this system is known to have a role in defensive reaction and anxiety (Lyonfields et al., 1995). Also, importantly, the tonic PD is unaffected by physical fitness factors (Filipe et al., 2003) and body mass (Piha et al., 1994; Filipe et al., 2003), which is not the case in other autonomic measures, such as heart rate and blood pressure (Gelber et al., 1997; Rennie et al., 2003). Accordingly, individual differences in physical capability affect tonic PD to a lesser extent than in other arousal measures.

There is very little data on whether PD is a reliable measure of tonic arousal. To clarify this issue, we conducted a pilot study where we measured the mean pupil size of 26 student participants for $1 \mathrm{~min}$ during rest, on two consecutive days. The test-retest correlation between the different measurements was high $(r=0.83$, $p<0.01$ ), supporting the reliability of the PD as a measure of tonic arousal.

\section{STUDY 1: LOSS VERSUS GAIN DOMAIN RISKS}

In this study we focused on two choice problems, one involving risks in the gain domain and the other involving risks in the loss domain. The two choice problems were as follows:

Problem 1. Gain Condition:

$S \quad 600$ with certainty

$R \quad 0$ or 1200 with equal probability $\quad P(R)=0.38$
Problem 2. Loss Condition:

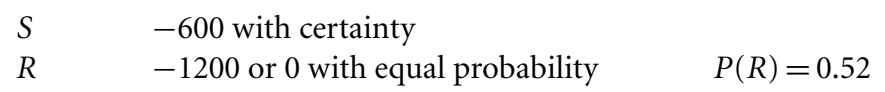

In each choice problem, the participants selected among two alternatives with equal expected value, a safe alternative $(S)$ and a riskier alternative $(R)$. These two problems are identical but for fact that in the Loss condition all outcomes are multiplied by $-1 . P(R)$ denotes the average proportion of $R$ selections in Study 1 .

The two choice problems were administered in an experiencebased task. The participants were not provided with a written description of the payoffs but rather had to learn to make choices by sampling the alternatives and receiving feedback. The task began with the participant facing two blank buttons. Upon selecting a button a payoff was sampled from the alternative's payoff distribution and the participant was presented with this payoff as feedback. The use of experience-based tasks is common in studies of physiological arousal and brain activity (e.g., Bechara et al., 1997). Risk taking in these experiential tasks is typically operationalized as the average proportion of selections from the alternative with the higher variance payoffs (see Yechiam and Ert, 2011). Our main prediction was that the association between tonic pupil size (as recorded prior to the task) and risk taking level would be more pronounced in the Loss condition.

\section{METHOD \\ Participants}

The participants were 20 undergraduates from the Technion (12 men and 8 women). Their average age was 23 (ranging from 20 to 27). Upon completing the experiment, they were given a fixed fee which was updated according to the accumulated amount of tokens won (or lost) in the experimental task. The conversion rate of experimental tokens to money was 1 Israeli Shekel per 1000 point earned (participants were informed of the conversion rate beforehand).

\section{Behavioral task}

All participants completed two experience-based decision tasks involving 100 trials. Half of them performed the Loss condition task followed by the Gain condition task and the others performed the tasks in reverse order. In both tasks, they were asked to operate a "money machine" with two choice alternatives presented as blank virtual buttons (see Hertwig et al., 2004; Newell and Rakow, 2007; Erev and Haruvy, in press). They were informed simply that their task would be to repeatedly select a button in the machine in order to maximize their total earning. They were further told that they would perform two tasks but their final payoffs would be set according to the accumulating amount from one of the tasks, selected at random. This was done to prevent diversification biases and income effects across different tasks (Cho and Luce, 1995).

Each button selection was followed by the presentation of the obtained payoff and the accumulated payoff, allowing participants to learn to select the choice alternatives from their experience. The outcomes from the two buttons were drawn 
from the payoff distributions for the Gain and Loss condition problems presented above. The allocation of the Safe and Risky alternative to the left and right buttons was randomized for each participant, but was kept constant throughout an experimental condition (e.g., in the Gain condition). The outcomes were randomly generated on each trial from the alternative's payoff distribution.

\section{PD measure}

Pupillometry data was collected using ViewPoint PC 60 EyeFrame system (Arrington Research, Scottsdale, Arizona). The system operates with a single tiny camera and an infrared illuminator mounted on a lightweight frame facing toward the participant's dominant eye, and supported by comfortable head straps. It records pupil data at approximately 30 frames per second (fps). Pre-task pupil size samples were taken just after the calibration and prior to the start of the decision-making task for a period of $30 \mathrm{~s}$. During this time the participants looked at the center of the screen and were asked to wait for the experimenter's instructions. The eye tracking measures continued while the participants performed the decision task.

Like other autonomic measures (e.g., GSR) the tonic pupil size is affected by gender, with some studies demonstrating larger pupil sizes for women than men (Zinn, 1972; Alexandridis, 1985) though this is disputed (see Jones, 1990; Filipe et al., 2003). Accordingly, we examined the possible effect of gender on the pre-task PD, as well as on risk taking levels.

\section{Design and analysis}

Our main prediction pertained to the negative association between pre-task arousal and risk taking in the two within-subject task conditions. We therefore used a one-tailed test for this analysis (as explicitly noted in the results below). For all other analyses, we used two-tailed tests.

\section{RESULTS AND DISCUSSION \\ Risk taking and pupil diameter during the task}

The average proportion of risky selections in the Gain condition was $0.38(\mathrm{SD}=0.23)$, while in the Loss condition it was 0.52 $(\mathrm{SD}=0.24)$. Thus, the findings show greater risk seeking with losses. This pattern is similar to the reflection effect observed by Kahneman and Tversky's (1979). An examination of the median behavior showed more distinct changes in risk taking as a function of the payoff domain, with 0.4 selections of the risky alternative in the Gain condition and 0.63 in the Loss condition. This difference between conditions was statistically significant $[t(19)=2.38$, $p=0.03]$. The correlation between $P(R)$ in the two conditions was $r=0.37, p=0.10$; as found previously (Ert and Yechiam, 2010; Yechiam and Ert, 2011) participants had a somewhat consistent tendency to prefer certainty to risk or vise versa, though it was not statistically significant.

Although our main predictions pertained to pre-task PD, we also measured the PD during the task. The results are shown in Figure 1. Consistent with previous studies (e.g., Satterthwaite et al., 2007; Hochman and Yechiam, 2011) the participants' PD was higher following losses (of -1200 or -600) compared to equivalent gains. The difference between the PD following losses

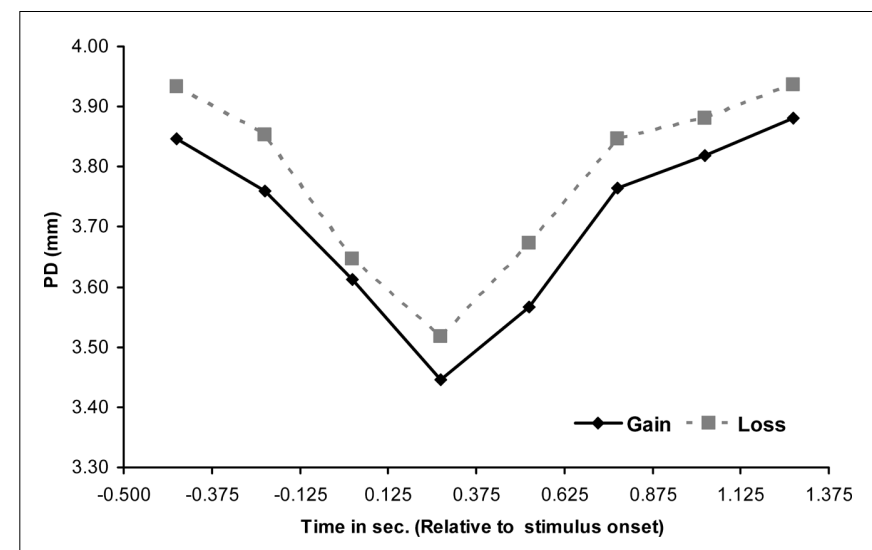

FIGURE 1 | Mean pupil diameter in the Loss and Gain conditions (in $\mathrm{mm}$ ) across participants and events (of loss: -600 or -1200 , or of gain: 600 or 1200). Time zero denotes the outcome presentation onset.

and gains was significant $0.375-0.875 \mathrm{~s}$ following the outcome presentation $[t(19)=2.71, p=0.04]^{2}$.

To examine the effect of losses on extended arousal we also compared the response time in the Gain and Loss conditions (see Porges, 1992). Previously, RTs were found to be longer in loss compared to gain domain tasks (e.g., Yechiam and Telpaz, in press). The average RT in the Loss condition was $0.61 \mathrm{~s}$ while in the Gain condition it was $0.46 \mathrm{~s}$. The difference was significant $[t(19)=2.52$, $p=0.02]$. Thus, losses seemed to increase both immediate arousal and processing time beyond gains.

\section{Pre-task pupil diameter}

Scatter plots of risk taking proportions by pre-task PD appear in Figure 2. As clearly indicated in the figure, individuals with high pre-task PD tended to make fewer risky selections in the Loss condition, but this was much less evident in the Gain condition. In the Loss condition the correlation between pretask PD and $P(R)$ was $r=-0.44, p=0.026$ (one tailed). In the gain condition the correlation was only $r=-0.25, p=0.15$ (one tailed).

To verify that this result is not due to women participants having larger PDs (as found previously; e.g., Alexandridis, 1985) and also taking less risk, we examined gender differences in the studied variables. The pre-task PD in women (Mean $=3.62 \mathrm{~mm}, \mathrm{SD}=0.40$ ) was actually smaller than that of the men $($ Mean $=4.26 \mathrm{~mm}, \mathrm{SD}=0.40)$, but the difference did not reach significance $t(18)=1.69, p=0.11$. Additionally, there were no significant gender differences in the proportion of risky selections in the Loss and Gain conditions. We can thus rule out gender as leading to major differences in the studied variables.

The results are consistent with our prediction that tonic arousal - risk taking associations should be stronger in the Loss condition compared to the Gain condition. The direction of the effect is in turn consistent with the prediction of the

\footnotetext{
${ }^{2}$ Note that as shown in Figure 1 there was a (non-significant) difference in PD before obtaining losses and gains. This is to be expected given the fact that the participants knew they were about to face losses.
} 

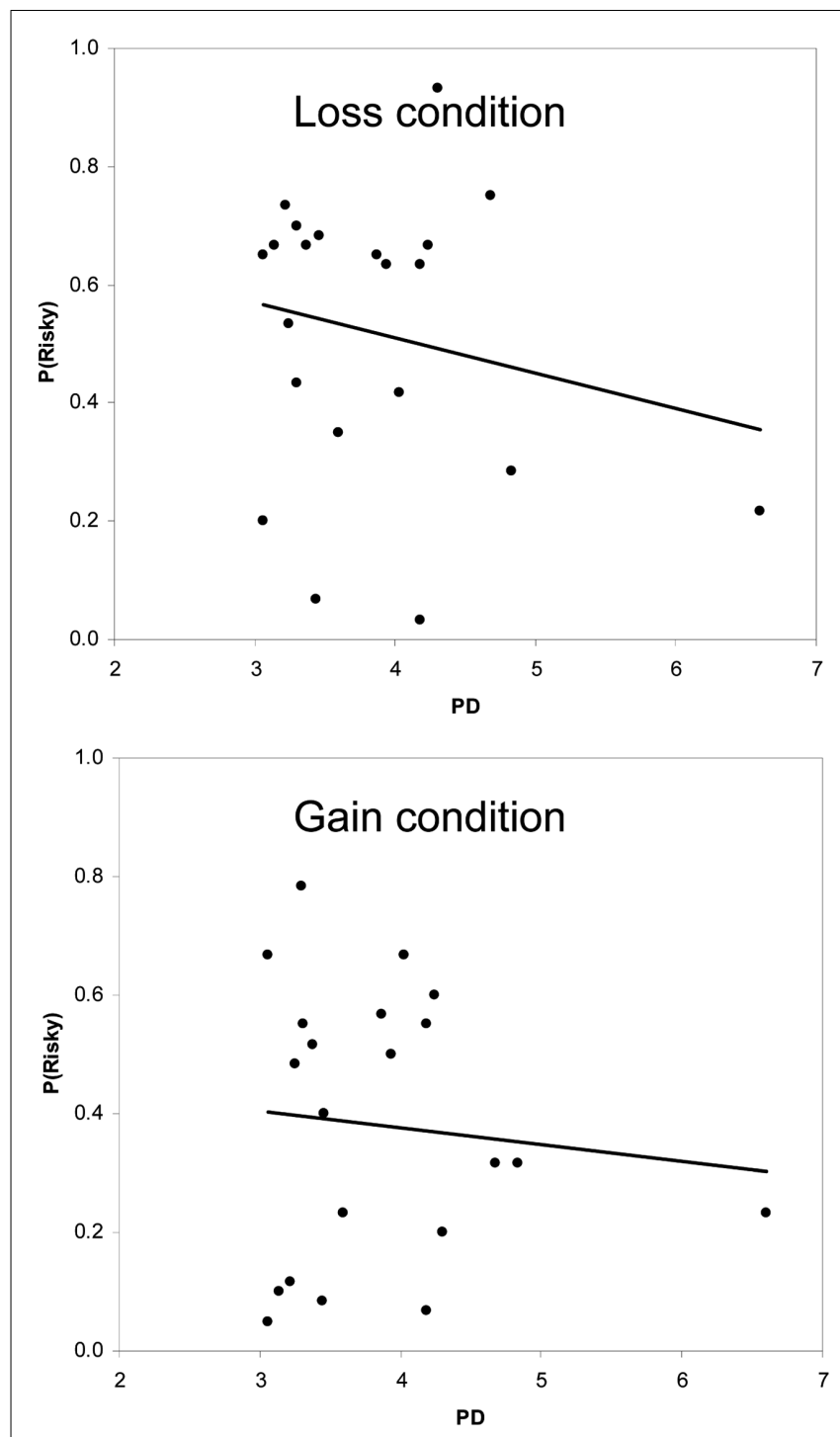

FIGURE 2 | Scatter plot and regression lines of the proportion of risky selections across $\mathbf{1 0 0}$ trials by pre-task PD in Study 1. Top: Loss condition. Bottom: Gain condition.

aforementioned personality theories: those showing low-arousal tended to take more risk. We have argued that this phenomenon is due to the attentional effects of losses. However, an alternative explanation is that it is due to loss aversion. If one assumes that (a) losses have greater subjective weight than gains, and (b) people behave more reliably in tasks that are of importance to them (Judd and Krosnick, 1989; Chaiken and Maheswaran, 1994; Kanfer et al., 1994), then it stands to reason that individuals would exhibit more reliable risk taking behavior in a loss-domain task. Loss aversion can therefore increase the association between tonic arousal and risk taking behavior. In our second study we therefore examined whether these effect of losses can be observed simultaneously with no loss aversion, namely with the participants showing no behavioral tendency to give greater weight to losses over gains.

\section{STUDY 2: MIXED GAINS AND LOSSES RISK}

In experience-based tasks it was previously found that people do not display loss aversion when the risk involves symmetric gains and losses (see Erev et al., 2008; Silberberg et al., 2008; Hochman and Yechiam, 2011; Yechiam and Telpaz, in press). While the generality of this finding into other situations is a matter or controversy (Rick, 2011), the fact that in the experiential setting there is no loss aversion provides a testing ground for whether different effects of losses may emerge even in the absence of a decision weight asymmetry of the sort proposed by Kahneman and Tversky (1979).

The study focused on the following two choice problems:

Problem 3. Gain Condition:

$S \quad 2$ or 4 with equal probability

$R \quad 1$ or 5 with equal probability $\quad P(R)=0.49$

Problem 4. Mixed Condition:

$S \quad-1$ or 1 with equal probability

$R \quad-2$ or 2 with equal probability $\quad P(R)=0.54$

The term Mixed refers to a choice problem producing both gains and losses. In the studied Mixed condition the risky alternative produced gains and losses of the same probability and magnitude. Under loss aversion people should avoid risk in this situation. An existing pupillometry dataset (Hochman and Yechiam, 2011; Study 1) in which these two problems were administered was used. Previous analyses of the data focused only on phasic (i.e., on-task) arousal (Hochman and Yechiam, 2011). We tested whether the association between tonic pupil size and risk taking would be more pronounced in risks with losses, even while the participants were loss neutral.

\section{METHOD}

Participants

Twenty-five undergraduates from the Technion (13 men and 12 women) participated in the study. The participants were given a fixed fee and were also compensated according to the number of points earned in the experimental task, at a rate of 0.1 Israeli Shekel per 1 point earned (they were informed of the conversion rate beforehand).

\section{Behavioral task}

All participants completed two experience-based decision tasks involving 60 trials. Approximately half of them performed the Mixed condition task followed by the Gain condition task and the other half performed the tasks in reverse order. The instructions were as in Study 1. The tasks involved operating a "money machine" with two choice alternatives. The payoffs were drawn from the outcomes of the Gain and Mixed conditions above. In order to make the incentive structure less obvious, a constant of $0.1-0.5$ (in 0.1 intervals) was randomly added or subtracted from the sampled payoff in every trial. Additionally, to eliminate possible surprise effects that would be non-symmetric with respect to gains and losses, payoffs were delivered in a deterministic fashion. Each choice alternative initially produced either a gain/relativegain or a loss/relative-loss, which was switched to a payoff from 
the opposite domain on each selection. About half of the participants were presented with a gain/relative-gain in the first selection, while for the other participants this order was reversed.

\section{PD measure}

Pre-task pupil size was examined as in Study 1. Due to a technical problem we did not get pre-task results from two of the participants. Thus, all correlations with tonic PD are based on a sample size of 23 .

\section{RESULTS AND DISCUSSION}

As reported in Hochman and Yechiam (2011), the average proportion of risky selections in the Mixed condition was not significantly different from 0.5 (Mean $=0.54, \mathrm{SD}=0.15)$. Clearly, the participants did not avoid the alternative incurring larger losses. Additionally, the average proportion of risky selections in the Mixed condition and in the Gain condition (Mean $=0.49, \mathrm{SD}=0.23$ ) was quite similar, $t(24)=1.07, p=0.30$. Thus, the participants exhibited no loss aversion in this experience-based choice task.

Differently from Study 1, the correlation between risky choices in the two task conditions was close to zero $(r=-0.02, p=0.91)$. This is consistent with previous results showing that consistency across domains is impaired when there is no sure thing alternative (Yechiam and Ert, 2011).

Scatter plots of risk taking proportions by pre-task PD appear in Figure 3. As illustrated in the figure, individuals with high pre-task PD tended to take less risk in the Mixed condition. In the Gain condition a surprising reverse trend was observed: those with higher pre-task PD actually took more risk. Correlation analyses showed that in the Mixed condition the pre-task PD was negatively correlated with the proportion of risky choices, $r=-0.37, p=0.04$ (one tailed), while in the Gain condition, pre-task PD was positively correlated with risky choices, $r=0.47$, $p=0.02$.

We examined whether the positive effect found in the Gain condition is a product of boredom at the end of the task. Previous findings suggest that in monotonous tasks that have little cognitive requirement, individuals with moderate levels of arousal seek to maintain their arousal (Fischer et al., 2008). One way to increase arousal may be to explore both alternatives and thus to also take risk (see Iglesias-Parro et al., 2001). Possibly, this situation was more likely to develop in the Gain condition because losses are more arousing. Also, recall that the task in Study 2 was more monotonic than in Study 1 because of the gainloss-gain-loss pattern. In the first half of the task, the negative correlation for the Mixed condition remained about the same, $r=-0.41, p=0.05$. By contrast, the positive correlation in the Gain task was found to be non-significant, $r=0.30, p=0.17$. Thus, consistent with the boredom-based explanation, the positive correlation for the Gain condition emerged only in the second half of the task (Gain: $r=43, p=0.04$; Mixed: $r=-0.40$, $p=0.06)$. For both halves of the task, the negative correlation predicted by personality theories was only apparent when the risk involved losses, and this was found simultaneously with no loss aversion.

We also examined gender differences in the studied variables. The pre-task PD in women $($ Mean $=4.59 \mathrm{~mm}, \mathrm{SD}=1.59)$ was
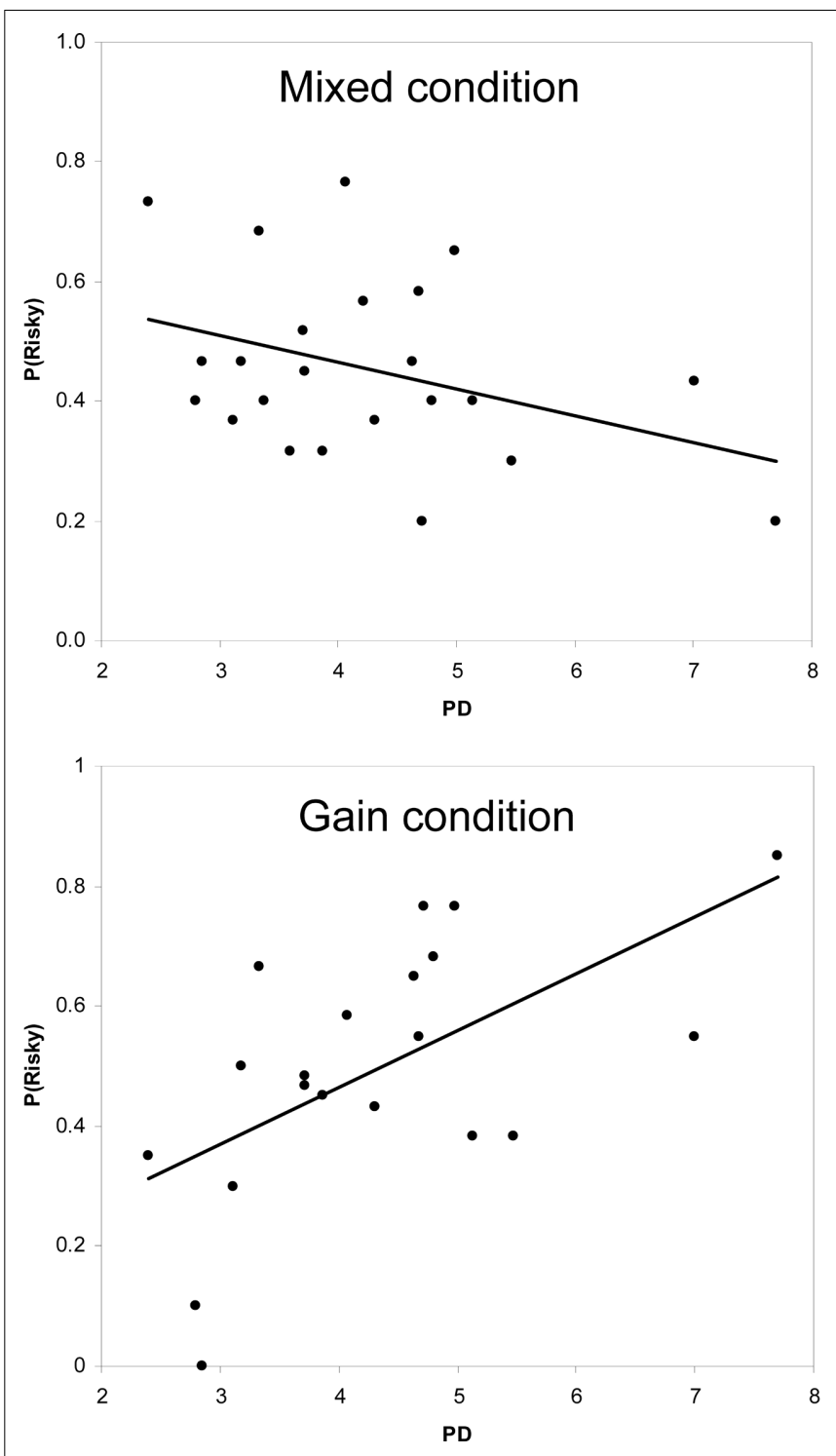

FIGURE 3 | Scatter plot and regression lines of the proportion of risky selections across trials by pre-task PD in Study 2. Top: Mixed condition. Bottom: Gain condition.

larger than that of the men $($ Mean $=3.92 \mathrm{~mm}, \mathrm{SD}=0.85)$ but not in a significant manner, $t(21)=1.24, p=0.22$. Additionally, there were no significant gender differences in the proportion of risky selections in the Mixed and Gain conditions. In fact, when aggregating the results across the two studies, the PD for both genders was quite similar (mean difference of $0.09 \mathrm{~mm}$ ).

\section{STUDY 3: REPLICATION USING MIXED GAINS AND LOSSES}

To examine the interpretation that the positive correlation in the Gain condition was a product of low task demands, we replicated Study 2 with time pressure and a secondary requirement to perform an arithmetic task between choice trials. We expected to replicate the negative correlation observed in Study 2 for risky losses, but not the positive correlation observed for risky gains. 


\section{METHOD}

\section{Participants}

Forty undergraduate students from the Technion (20 women and 20 men) participated in the experiment. Their average age was 24 (ranging from 19 to 28). Participants were given a fixed fee and were also compensated according to the number of points earned in the experimental task, with a conversion rate of 0.1 Israeli Shekel per 1 point earned.

\section{Behavioral task}

The decision task consisted of two buttons and two counters, as in Study 2. Participants performed 30 trials in each of the two experimental conditions (Mixed and Gain). The order of the Mixed and Gain task conditions was counter-balanced so that half of the participants performed each task first. The choice outcomes were as in Study 2. The participants received no prior information concerning the choice outcomes, and learned to make choices from their experience. An arithmetic task was administered along with the decision task, as follows.

In each trial participants made their decision and afterward performed an arithmetic task. They were required to accomplish both tasks in $7.5 \mathrm{~s}$, and failure to do so resulted in a loss of three points. The arithmetic problems were presented in the upper section of the screen. The answers were typed using a virtual keypad. The task involved adding or multiplying a single-digit number with a twodigit number. The exact numbers were randomly generated in each trial (the single-digit number was chosen from a uniform distribution ranging from 2 to 6 , and the two-digit number was chosen from a uniform distribution ranging from 10 to 17). If participants answered the arithmetic question incorrectly they received an "incorrect answer" feedback but could give other answers within the $7.5 \mathrm{~s}$ time window. The task included a time meter which noted the time left until the end of each trial. When participants answered the arithmetic problem correctly the screen elements of that task were darkened. Each participant was randomly allocated to receive either addition or multiplication questions. As the allocation of the participants into the addition or multiplication questions had no effect on the studied correlations, we pooled across this manipulation.

\section{PD measure}

Pre-task pupil size was examined as in Study 1. Due to a technical problem we did not get pre-task results from one of the participants, leaving a total of 39 participants.

\section{RESULTS AND DISCUSSION}

We first examined the difference between risk taking patterns in the Mixed and Gain conditions. As in Study 2, the proportion of risky selections in the two task conditions was quite similar, $0.46(\mathrm{SD}=0.19)$ in the Mixed condition and 0.46 in the Gain condition $(\mathrm{SD}=0.25), t(37)=0.02, p=0.98$. Scatter plots of risk taking proportions by pre-task arousal levels (in PD) appear in Figure 4. In the Mixed condition the participants' pre-task PD was negatively correlated with the proportion of risky selections ( $r=-0.34, p=0.02$, one tailed). However, in the Gain condition there was no significant correlation between the two measures ( $r=0.07, p=0.33$, one tailed). Thus, the positive association in

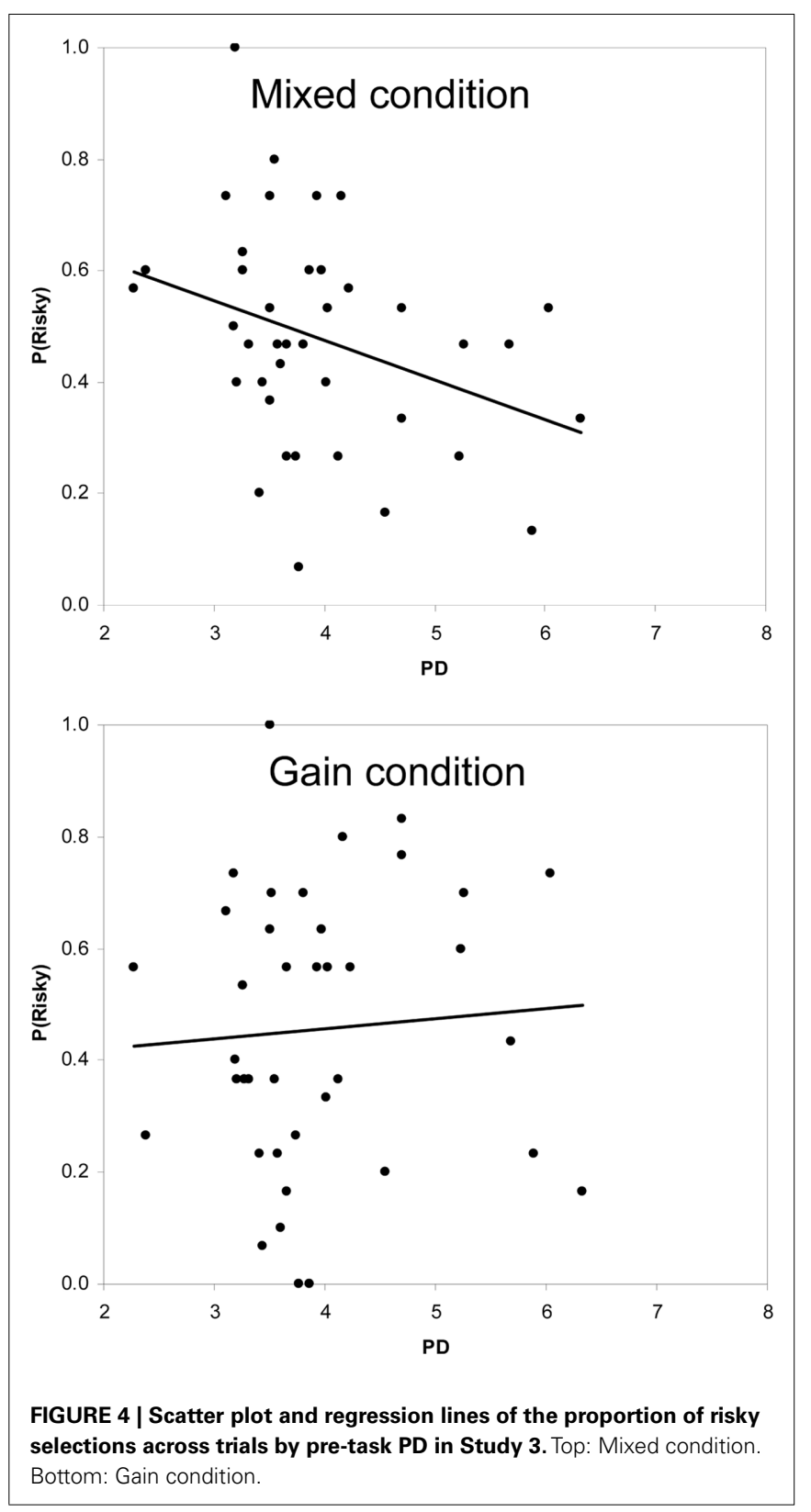

the Gain condition found in Studies 1 and 2 did not emerge in a statistically significant manner in Study $3^{3}$.

To sum up, in this study where the decision task was performed with moderate time pressure and with an additional cognitive requirement, we replicated the negative correlation between tonic PD and risk taking with losses. However, as opposed to Study 2, there was no positive association between arousal and risk taking in the gain domain. The findings are therefore consistent with the prediction of the attentional model of losses, which implies that

\footnotetext{
${ }^{3}$ An examination of possible gender effects revealed that the pupil size of women (Mean $=3.83 \mathrm{~mm}$., $\mathrm{SD}=0.83$ ) was not significantly different from that of the men [Mean $=4.02 \mathrm{~mm}$., $\mathrm{SD}=1.08], t(37)=0.59, p=0.56$. There were also no significant gender differences in risk taking both in the Mixed and Gain conditions.
} 
losses do not reverse the association between tonic PD and risk taking but rather, accentuate it.

\section{GENERAL DISCUSSION}

Several theories of personality and traits predict a negative association between arousal level and risk taking (e.g., Zuckerman et al., 1964; Gray, 1987; Zuckerman, 1990; Eysenck, 1992). Though the exact mechanisms that lead to this negative correlation are different in each theory, their general idea is similar: they suggest that people exhibiting lower levels of internal arousal seek stimulation by taking risk. Here, we hypothesized that the negative association between tonic arousal and risk taking would be enhanced in risks with losses, due to the simple fact that losses increase arousal and on-task attention (Yechiam and Hochman, 2011).

The results of our three pupillometry studies supported the predicted moderating effect of losses. In Study 1 we have shown that in a loss-domain task there was a significant negative correlation between pre-task PD and the proportion of risky selections. In an equivalent gain domain task this negative correlation was much lower and not statistically significant. In Study 2 we examined whether these effects of losses on arousal-behavior relationship are due to loss aversion. For this purpose we studied a previous pupillometry database (Hochman and Yechiam, 2011) which included a choice task with symmetric gains and losses, and an equivalent task in the gain domain. While the participants showed no loss aversion on average, a negative correlation between pretask arousal and risk taking was only observed in the condition with losses.

Somewhat surprisingly, in this second study we found that across all trials there was a positive correlation between pretask arousal and risk taking in the gain domain. We argued, however, this positive association did not represent a general pattern. This was supported by a block by block analysis, which showed that the positive association only appeared in the second half of the task. We explicitly examined this assertion in Study 3, where we showed that administering the decision task in a more demanding environment (involving time pressure and a secondary task) eliminated the positive correlation in the gain domain. In this study, as in Study 1, in the absence of losses there was a zero correlation between arousal and risk taking, and the addition of losses produced a negative correlation between these measures.

We proposed that the negative correlation between tonic arousal and risk taking in the conditions with losses reflects the effect of losses on arousal and attention. This explanation was supported by our findings that losses led to significantly elevated pupil size and response time compared to equivalent gains during the decision task. Our results are consistent with past findings on the effect of losses on arousal (e.g., Hochman and Yechiam, 2011).

Taken together, the current results suggest that losses are an inherent part of what makes things risky. Indeed, the dictionary definition of risk equates it with loss. For example, In Merriam Webster (2011) risk's first definition is "possibility of loss or injury (peril)." Similarly, the Oxford English Dictionary (OED, 1982) defines risk as "hazard, danger, exposure to mischance, or peril." Indeed, while there is some disagreement about the ancient origin of the word, it is considered to have come to the English language from the French word risque, and in this language it was adapted from Italian risco, which stands for "navigating among dangerous rocks" (Timmerman, 1986). In Hebrew the word "sikun" which denotes risk is derived from sakana, or danger. The economic definition of risk, which is based on the variance of the outcomes and is almost universally accepted as a way of operationally defining risk, ignores the relation between risk and losses.

Here we have shown that losses matter. When individuals with low tonic arousal take risk they only do so for risks that involve losses. Therefore, in adjusting their behavioral responses to their arousal states, people "acknowledge" things as risky only when losses are part of them. In risks that do not include losses, the link between arousal and risk taking is severed. We have also shown that this property of losses does not depend on loss aversion, and emerges in experience-based tasks, where typically and in our study as well, no loss aversion is exhibited (Erev et al., 2008). Thus, the studied effect of losses appears to be quite general. We have therefore suggested that it may be due to the effect of losses on arousal and attention, rather than due to an asymmetry in decision weights.

Our findings do not preclude, however, that there may be other factors which can serve as cues that a given situation is risky. Possibly, after a prolonged learning period individuals may learn to associate an alternative producing relative gains and relative-losses (or small gains) as risky. Yet in our study this did not happen in the course of 100 repeated choice trials. Factors such as the size of the relative-loss could play a part in this. Another important limitation of the current studies is the small sample sizes used. This issue is especially pertinent in the context of examining individual differences (see e.g., Stanovich and West, 2008).

The current findings may explain some of the mixed findings in the literature on the association between physiological indices of tonic arousal and sensation seeking. Gerra et al.'s (1999) study of healthy adults demonstrated a positive association between NE concentration and sensation seeking on Zuckerman et al.'s (1964) scale, whereas similar studies of clinical populations reported negative correlations (Ballenger et al., 1983; Arque et al., 1988; Zuckerman, 1994). Studies of tonic endocrine levels in pathological gamblers also obtained mixed results (Ramirez et al., 1988; Roy et al., 1988; Schmitt et al., 1998). The Sensation Seeking Scale is a list of activities (e.g., "I would like to take the sport of water skiing," "I would not like to learn to fly an airplane") without explicit information concerning the perceived outcomes of those activities. Possibly, some individuals find these activities more dangerous; and this activates the negative association between tonic arousal and risk taking. Yet for others who find these activities less dangerous, this may not occur.

The current findings may also be relevant to the issue of risk communication. The experimental results suggest that presenting the negative side effect of risky products (e.g., cigarets) is a double edged sword. On the one hand, it reduces the attractiveness of the risky alternative; but on the other hand it may increase the arousal associated with it and this can actually attract some individuals.

\section{ACKNOWLEDGMENTS}

This research was supported in part by the European Commission Integrated Project IP-SKILLS-35005. 


\section{REFERENCES}

Alexandridis, E. (1985). The Pupil. New York: Springer-Verlag.

Arque, J. M., Unzeta, M., and Torrubia, R. (1988). Neurotransmitter systems and personality measurement: a study in psychosomatic patients and healthy subjects. Neuropsychobiology 19, 149-157.

Ballenger, J. C., Post, R. M., Jimerson, D. C., Lake, C. R., Murphy, D., Zuckerman, M., and Cronin, C. (1983). Biochemical correlates of personality traits in normals: an exploratory study. Pers. Individ. Dif. 4 615-625.

Bechara, A., Damasio, H., Tranel, D., and Damasio, A. R. (1997). Deciding advantageously before knowing the advantageous strategy. Science 275, 1293-1295.

Bereby-Meyer, Y., and Erev, I. (1998). On learning to become a successful loser: a comparison of alternative abstractions of learning processes in the loss domain. J. Math. Psychol. 42, 266-286.

Chaiken, S., and Maheswaran, D. (1994). Heuristic processing can bias systematic processing: effects of source credibility, argument ambiguity, and task importance on attitude judgment. J. Pers. Soc. Psychol. 66, 460-473.

Cho, Y., and Luce, R. D. (1995). Tests of hypotheses about certainty equivalents and joint receipt of gambles. Organ. Behav. Hum. Decis. Process. 64, 229-248.

Coombs, C. H., and Lehner, E. P. (1981). Evaluation of two alternative models of a theory of risk: I. Are moment of distributions useful in assessing risk? J. Exp. Psychol. Hum. 7, 1110-1123.

Coombs, C. H., and Lehner, E. P. (1984). Conjoint design analysis of the bilinear model: an application to judgments of risk. J. Math. Psychol. 28, $1-42$.

Duxbury, D., and Summers, B. (2004). Financial risk perception: are individuals variance averse or loss averse? Econ. Lett. 84, 21-28.

Ellis, L. (1987). Relationships of criminality and psychopathy with eight other apparent behavioral manifestations of sub-optimal arousal. Pers. Individ. Dif. 8, 905-925.

Erev, I., Bereby-Meyer, Y., and Roth, A. (1999). The effect of adding a constant to all payoffs: experimental investigation, and implications for reinforcement learning models. J. Econ. Behav. Organ. 39, 111-128.

Erev, I., Ert, E., and Yechiam, E. (2008). Loss aversion, diminishing sensitivity, and the effect of experience on repeated decisions. J. Behav. Decis. Mak. 21, 575-597.
Erev, I., and Haruvy, E. (in press). "Learning and the economics of small decisions," in The Handbook of Experimental Economics, Vol. 2, eds J. H. Kagel and A. E. Roth (Princeton, NJ: Princeton University Press).

Ert, E., and Yechiam, E. (2010). Consistent constructs in individuals' risk taking in decisions from experience. Acta Psychol. (Amst.) 134, 225-232.

Eysenck, H. (1967). The Biological Basis of Personality. Springfield, IL: Thomas.

Eysenck, M. W. (1992). Anxiety. The Cognitive Perspective. Hove: Erlbaum.

Farley, F. H., and Farley, S. V. (1972). Stimulus-seeking motivation and delinquent behavior among institutionalized delinquent girls. J. Consult. Clin. Psychol. 39, 94-97.

Filipe, J. A. C., Falcao-Reis, F., CastroCorreia, J., and Barros, H. (2003). Assessment of autonomic function in high level athletes by pupillometry. Auton. Neurosci. 104, 66-72.

Fischer, T., Langner, R., Birbaumer, N., and Brocke, B. (2008). Arousal and attention: Self-chosen stimulation optimizes cortical excitability and minimizes compensatory effort. $J$. Cogn. Neurosci. 8, 1443-1453.

Gatzke-Kopp, L. M., Raine, A., Loeber, R., Stouthamer-Loeber, M., and Steinhauer, S. R. (2002). Serious delinquent behavior, sensation seeking, and electrodermal arousal. J. Abnorm. Child Psychol. 30, 477-486.

Gelber, D. A., Pfeifer, M., Dawson, B., and Schumer, M. (1997). Cardiovascular autonomic nervous system tests: determination of normative values and effect of confounding variables. J. Auton. Nerv. Syst. 62, 40-44.

Gerra, G., Avanzini, P. Zaimovic, A., Sartori, R., Bocchi, C., Timpano, M., Zambelli, U., Delsignore, R. Gardini, F. Talarico, E., and Brambilla, F. (1999). Neurotransmitters, neuroendocrine correlates of sensation-seeking temperament in normal humans. Neuropsychobiology 39, 207-213.

Granholm, E., and Steinhauer, S. R. (2004). Pupillometric measures of cognitive and emotional processes. Int. J. Psychophysiol. 52, 1-6.

Gray, J. A. (1987). The Psychology of Fear and Stress. New York: Cambridge University Press.

Hertwig, R., Barron, G., Weber, E., and Erev, I. (2004). Decisions from experience and the effect of rare events in risky choice. Psychol. Sci. 15, 534-539.

Hochman, G., Glockner, A., and Yechiam, E. (2010). "Physiological measures in identifying decision strategies," in Foundations for tracing intuitions, challenges, and methods, eds A. Glockner and C. Witteman (London: Psychology Press and Routledge), 139-159.

Hochman, G., and Yechiam, E. (2011) Loss aversion in the eye and in the heart: the autonomic nervous system's responses to losses. J. Behav. Decis. Mak. 24, 140-156.

Hutchins, J. B., and Corbett, J. J. (1997). "The visual system," in Fundamental Neuroscience, ed. D. E. Haines (New York: Churchill Livingstone), 265-284.

Iglesias-Parro, S., Ortega, A. R., De la Fuente, E. I., and Martin, I. (2001). Context variables as cognitive effort modulators in decision making using an alternative-based processing strategy. Qual. Quant.35, 311-323.

Jones, R. (1990). Do women and myopes have larger pupils? Invest Ophthalmol. Vis. Sci. 31, 1413-1415.

Judd, C. M., and Krosnick, J. A. (1989). "The structural bases of consistency among political attitudes: effects of political expertise and attitude importance," in Attitude Structure and Function, eds A. R. Pratkanis, S. J. Breckler, and A. G. Greenwald (Mahwah, NJ: Lawrence Erlbaum), 99-128.

Kahneman, D., and Tversky, A. (1979). Prospect theory: an analysis of decision under risk. Econometrica 47, 263-291.

Kanfer, R., Ackerman, P. L., Murtha, T. C., Dugdale, B., and Nelson, L. (1994). Goal setting, conditions of practice, and task performance: a resource allocation perspective. J. Appl. Psychol. 79, 826-835.

Lyonfields, J. D., Borkovec, T. D., and Thayer, J. F. (1995). Vagal tone in generalized anxiety disorder and the effects of aversive imagery and worrisome thinking. Behav. Ther. 26 457-466.

Markowitz, H. M. (1952). Portfolio selection. J. Finance 7, 77-91.

McNamara, L., and Ballard, M. E. (1999). Resting arousal, sensation seeking and music preference. Genet. Soc. Gen. Psychol. 125, 229-250.

Newell, B. R., and Rakow, T. (2007). The role of experience in decisions from description. Psychon. Bull. Rev. 14, 1133-1139.

Piha, S. J., Ronnemaa, T., and Koskenvuo, M. (1994). Autonomic nervous system function in identical twins discordant for obesity. Int. J. Obes. 18, 547-550.

Plouffe, L., and Stelmack, R. M. (1986). Sensation-seeking and the electrodermal orienting response in young and elderly females. Pers. Individ. Dif. 7, 119-120.

Porges, S. W. (1992). "Autonomic regulation and attention," in Attention and Information Processing in Infants and Adults, eds B. A. Campbell, $H$. Hayne, and R. Richardson (Hillsdale, NJ: Lawrence Erlbaum), 201-226.

Pratt, J. W. (1964). Risk aversion in the small and in the large. Econometrica 32, 122-136.

Preuschoff, K., Bossaerts, P., and Quartz, S. R. (2006). Neural differentiation of expected reward and risk in human subcortical structures. Neuron 51, 381-390.

Preuschoff, K., Quartz, S. R., and Bossaerts, P. (2008). Human insula activation reflects risk prediction errors as well as risk. J. Neurosci. 28, 2745-2752.

Ramirez, L. F., McCornick, R. A., and Lowy, M. T. (1988). Plasma cortisol and depression in pathological gamblers. Br. J. Psychiatry 153, 684-686.

Rennie, K. L., Hemingway, H., Kumari, M., Brunner, E., Malik, M., and Marmot, M. (2003). Effects of moderate and vigorous physical activity on heart rate variability in a British study of civil servants. Am. J. Epidemiol. 158, 135-143.

Rick, S. (2011). Losses, gains, and brains: neuroeconomics can help to answer open questions about loss aversion. J. Consum. Psychol. 21, 453-463.

Roy, A., Adinoff, B., Roehrich, L., Lamparski, D., Custer, R., Lorenz, V., Barbaccia, M., Guidotti, A., Costa, E., and Linnoila, M. (1988). Pathological gambling: a psychobiological study. Arch. Gen. Psychiatry 45, 369-373.

Rozin, P., and Royzman, E. B. (2001). Negativity bias, negativity dominance, and contagion. Pers. Soc. Psychol. Rev. 5, 269-320.

Satterthwaite, T. D., Green, L., Myerson, J., Parker, J., Ramaratnam, M., and Buckner, R. L. (2007). Dissociable but inter-related systems of cognitive control and reward during decision making: evidence from pupillometry and event-related fMRI. Neuroimage 37, 1017-1031.

Schmitt, L. H., Harrison, G. A., and Spargo, R. M. (1998). Variation in epinephrine and cortisol excretion rates associated with behavior in an Australian Aboriginal Community. Am. J. Phys. Anthropol. 106, 249-253.

Sharpe, W. F. (1964). Capital asset prices: a theory of market equilibrium under conditions of risk. J. Finance 19, 425-442. 
Silberberg, A., Roma, P. G., Huntsberry, M. E., Warren-Boulton, F. R., Takayuki, S., Ruggiero, A. M., and Suomi, S. J. (2008). On loss aversion in capuchin monkeys. J. Exp. Anal. Behav. 89, 145-155.

Stanovich, K. E., and West, R. F. (2008). On the relative independence of thinking biases and cognitive ability. J. Pers. Soc. Psychol. 94, 672-695.

Stelmack, R. M., Plouffe, L., and Falkenberg, W. (1983). Extraversion, sensation-seeking and electrodermal response: Probing a paradox. Pers. Individ. Dif. 4, 607-614.

Taylor, S. E. (1991). The asymmetrical impact of positive and negative events: the mobilizationminimization hypothesis. Psychol. Bull. 110, 67-85.
Timmerman, P. (1986). "Methodology and surprise in the sustainable development of the biosphere," in Sustainable Development of the Biosphere, eds W. Clark and R. Munn (Cambridge: Cambridge University Press), 435-444.

Yechiam, E., and Ert, E. (2011). Risk attitude in decision making: in search of trait-like constructs. Top. Cogn. Sci. 3, 166-186.

Yechiam, E., and Hochman, G. (2011). Loss-aversion or loss-focusing: the positive impact of losses on performance and the tax-max effect. (Manuscript submitted for publication).

Yechiam, E., and Telpaz, A. (in press). Losses induce consistency in risk taking even without loss aversion. J. Behav. Decis. Mak.
Zinn, K. E. (1972). The Pupil. Springfield, IL: Charles C. Thomas.

Zuckerman, M. (1960). The development of an affect adjective check list for the measurement of anxiety. $J$. Consult. Psychol. 24, 457-462.

Zuckerman, M. (1990). The psychophysiology of sensation seeking. J. Pers. 58, 313-345.

Zuckerman, M. (1994). Behavioral Expression and Biosocial Bases of Sensation Seeking. New York: Cambridge University Press.

Zuckerman, M., Kolin, E. A., Price, L. and Zoob, I. (1964). Development of a sensation seeking scale. J. Consult. Psychol. 28, 477-482.

Conflict of Interest Statement: The authors declare that the research was conducted in the absence of any commercial or financial relationships that could be construed as a potential conflict of interest.

Received: 18 September 2011; paper pending published: 17 October 2011; accepted: 03 November 2011; published online: 22 November 2011.

Citation: Yechiam E and Telpaz A (2011) To take risk is to face loss: a tonic pupillometry study. Front. Psychology 2:344. doi: 10.3389/fpsyg.2011.00344

This article was submitted to Frontiers in Cognitive Science, a specialty of Frontiers in Psychology.

Copyright (c) 2011 Yechiam and Telpaz. This is an open-access article subject to a non-exclusive license between the authors and Frontiers Media SA, which permits use, distribution and reproduction in other forums, provided the original authors and source are credited and other Frontiers conditions are complied with. 